\title{
Modelagem de nicho ecológico de musgos bioindicadores
}

\author{
Anny Bianca Santos Cruz*®, Juliano Ricardo Fabricante®, Thieres Santos Almeida®, \\ Universidade Federal de Sergipe, Itabaiana, 49.510-200, Sergipe, Brasil. *bianca.bbb@live.com
}

Recebido: 7 novembro 2020 / Aceito: 08 fevereiro 2021 / Publicado online: 24 maio 2021

\begin{abstract}
Resumo
As briófitas podem ser utilizadas como bioindicadoras, no entanto, uma das limitações desse uso são as lacunas no conhecimento sobre a ocorrência das espécies. Assim, o presente estudo teve como objetivo realizar a modelagem de nicho ecológico para Helicodontium capillare (Hedw.) A.Jaeger e Thuidium tomentosum Schimp., duas espécies de musgos bioindicadores. Os dados de ocorrência das espécies foram obtidos na plataforma Global Biodiversity Information Facility (GBIF) e SpeciesLink. As variáveis bioclimáticas foram coletadas do WorldClim 2.0. Para selecionar as menos correlacionadas foi realizada a análise de correlação de Pearson. A modelagem foi feita utilizando os algoritmos Generalized Additive Models, Gaussian Process, MaxEnt, Random Forest e Support Vector Machine. Usando os melhores modelos foi gerado um modelo consenso. A validade dos modelos foi testada utilizando a métrica True Skill Statistic (TSS). Os resultados indicam que as duas espécies apresentam maior adequabilidade ambiental em ambientes com alta precipitação principalmente em países industrializados da América Latina à exemplo do Brasil e Colômbia. Além disso, as áreas de maior adequabilidade também correspondem à grandes centros urbanos com altos índices de poluição. Portanto, essas espécies podem ser utilizadas como bioindicadoras nessas regiões, auxiliando assim no processo de identificação e gestão do problema.
\end{abstract}

Palavras-chave: Briófitas, susceptibilidade de ocorrência, contaminação ambiental, monitoramento ambiental.

\section{Ecological niche modeling of bioindicator mosses}

\begin{abstract}
Bryophytes can be used as bioindicators, however one limitations of this use is knowledge gaps about the occurrence of species. Thus, the present study aimed to perform the ecological niche modeling for Helicodontium capillare (Hedw.) A. Jaeger and Thuidium tomentosum Schimp., two species of bioindicator mosses. Species occurrence data were obtained from Global Biodiversity Information Facility (GBIF) and SpeciesLink platform. Bioclimatic variables were collected from WorldClim 2.0, to select the least correlated variables, Pearson's correlation analysis was performed. Modeling was performed using the Generalized Additive Models, Gaussian Process, MaxEnt, Random Forest and Support Vector Machine algorithms. Using best models, a consensus model was generated. The validity of the models was tested using the True Skill Statistic (TSS) metric. Results show that the two species have greater environmental suitability in environments with high rainfall, especially in industrialized countries in Latin America, such as Brazil and Colombia. In addition, the areas of greatest suitability also correspond to large urban centers with high levels of pollution. Therefore, these species can be used as bioindicators in these regions, thus assisting in the problem identification and management process.
\end{abstract}

Keywords: bryophytes, susceptibility to occurrence, environmental contamination, environmental monitoring.

\section{Introdução}

As briófitas representam o segundo maior grupo de plantas do planeta (Buck, 2000). Elas estão divididas em Bryophyta, Marchantiophyta e Anthocerotophyta (Goffinet \& Shaw, 2009). Desses, o grupo Bryophyta, formado pelos musgos, é o maior em número de espécies, apresentando cerca de 12.000 táxons (Vanderpoorten \& Goffinet, 2009). Já para o Brasil, são reconhecidas cerca de 3.125 espécies de briófitas (Shepherd, 2005).

As briófitas apresentam ampla ocorrência geográfica e são consideradas espécies colonizadoras (Vieira, Oliveira, Silva, Silva, da Conceição \& Oliveira, 2017). Além disso, muitas delas, principalmente musgos, costumam ser sensíveis a mudanças no meio ambiente (Frahm, 1991; Inácio-Silva, Carmo \& Peralta, 2017). Por conta destas características, espécies desse grupo podem ser utilizadas como bioindicadores das condições ambientais (Câmara, Teixeira, Lima \& Lima, 2003).

Dentre as condições que podem ser indicadas por táxons do grupo, está a contaminação por metais pesados (Mazzoni, Lanzer, Schafer, Bordin \& Wasum, 2012; Barbosa \& Carvalho, 2016). Esses elementos podem causar sérios problemas ao meio ambiente e a saúde humana (Muniz \& Oliveira-Filho, 2006; Bagliano, 2012; Teixeira, Moreira, 
Coelho, Amaral \& Cupertino, 2020), a exemplo de alterações genéticas, lesões cerebrais, problemas renais, diferentes tipos de câncer, dentre outros. Assim, é importante a detecção dos mesmos, para que dessa forma, possam ser realizadas ações de descontaminação dos ambientes.

As espécies Helicodontium capillare (Hedw.) A.Jaeger e Thuidium tomentosum Schimp. são alguns exemplos de briófitas que podem ser utilizadas para indicação de metais pesados no ambiente, a exemplo do zinco, níquel, cobre, chumbo e cromo (Mazzoni, et al., 2012). Contudo, antes é necessário determinar em quais localidades essas espécies podem ocorrer.

Desta forma, o uso da modelagem de nicho ecológico pode ser uma alternativa promissora. Essa análise prediz áreas com adequabilidade ambiental para ocorrência das espécies utilizando pontos de ocorrências conhecidos e dados ambientais (Guisan \& Zimmermann, 2000).

As pesquisas sobre briófitas no Brasil ainda são escassas, principalmente quando relacionadas a modelagem. Como exemplo podemos citar os estudos desenvolvidos por Silva, Kamino \& Pôrto, (2014) e Inácio-Silva et al. (2017).

Assim, o presente estudo teve como objetivo realizar a modelagem de nicho ecológico para $H$. capillare e $T$. tomentosum, duas espécies de musgos bioindicadores.

\section{Material e Métodos}

Foram coletados pontos de ocorrência georreferenciados das espécies selecionadas nas bases de dados online GBIF (GBIF, 2020) e SpeciesLink (CRIA, 2020). Os pontos duplicados, com erros (no oceano, coordenada nula, pontos duvidosos) e de centroides de municípios foram removidos. Ao final da coleta e filtragem de dados restaram 236 ocorrências para T. tomentosum e 207 para H. capillare.

Foram utilizadas as 19 variáveis bioclimáticas de temperatura e precipitação do WorldClim 2.0. Essas variáveis são resultantes de dados históricos dos anos de 1970 a 2000 (Fick \& Hijmans, 2017). Todas as variáveis utilizadas possuíam uma resolução de $2,5 \mathrm{~min}$. Para reduzir a colinearidade e selecionar as variáveis utilizadas no modelo, foi realizada a análise de correlação de Pearson entre as variáveis, com um valor de corte de 0,7 (Dormann et al., 2013).

Foram gerados pontos de pseudo ausência utilizando o método de "Enviromental constrain", onde as pseudoausências são alocadas em áreas de baixa adequabilidade ambiental preditas por um modelo Bioclim (Barbet-Manssin, Jiguet, Albert \& Thuiller, 2012; Andrade, Velazco \& Junior, 2020). O número de pseudo-ausências geradas obedeceu a razão 10 para um ponto de ocorrência (Barbet-Manssin et al., 2012), totalizando 2360 pseudo-ausências para T. tomentosum e 2070 para $H$. capillare.

Para o processo de modelagem foram utilizados os algoritmos Generalized Additive Models (GAM) (Guisan, Edwards \& Hastie, 2002), Gaussian Process (GAU) (Golding \& Purse, 2016), Maximum Entropy (MaxEnt) (Phillips, Anderson \& Schapire, 2006), Random Forest (RF) (Breiman, 2001) e Support Vector Machine (SVM) (Salcedo-Sanz, RojoÁlvarez, Martínez-Ramón \& Camps-Valls, 2014). Os modelos foram replicados 10 vezes em bootstrap utilizando $70 \%$ dos pontos para o treino e $30 \%$ para o teste (Zurell et al., 2020).

A validade dos modelos foi testada utilizando a métrica TSS (True Skill Statistic), onde valores próximos ou iguais a 1 indicam predição satisfatória e valores menores que 0 indicam que o modelo não é melhor que uma predição aleatória (Allouche Tsoar, \& Kadmon, 2006). No final foi gerado um modelo consensual através da técnica "ensemble" utilizando a média dos melhores modelos avaliados. Nessa técnica são selecionados os modelos que apresentaram valores de TSS maior que o valor médio obtido entre todos os modelos (Velazco, Villalobos, Galvão, \& Júnior, 2019). Todos os modelos foram gerados no pacote "ENMTML" (Andrade et al., 2020) no ambiente R (R CORE TEAM, 2020). O layout dos mapas foi posteriormente construído no QGIS.

\section{Resultados e Discussão}

A análise de correlação de Pearson indicou alta correlação entre a maior parte das variáveis, sendo selecionadas as variáveis: Bio2 - Variação Diurna Média de Temperatura, Bio3 - Isotermalidade, Bio8 - Temperatura média do trimestre mais úmido, Bio9 - Temperatura média do trimestre mais seco, Bio12 - Precipitação Anual, Bio15 Sazonalidade da Precipitação. Todos os modelos gerados foram validados com TSS > 0 com a maioria dos modelos atingindo valores de TSS >0,5. Os mapas consensuais obtiveram TSS $=0,65$ e 0,83 para as espécies $T$. tomentosum e H. capillare respectivamente (Tabela 1 ).

Tabela 1. Modelos de nicho ecológico para as espécies Thuidium tomentosum Schimp. e Helicodontium capillare (Hedw.) A.Jaeger com diferentes algoritmos e modelo consenso (Ensemble) dos modelos com TSS acima da média.

\begin{tabular}{lcccccc}
\hline \multicolumn{7}{c}{ T. tomentosum } \\
\hline Algoritmo & GAM & GAU & MaxEnt & RF & SVM & Ensemble \\
TSS & 0.317 & $0.521^{*}$ & 0.272 & $0.713^{*}$ & $0.479^{*}$ & 0.649 \\
\hline \multicolumn{7}{c}{ H. capillare } \\
\hline Algoritmo & GAM & GAU & MaxEnt & RF & SVM & Ensemble \\
TSS & 0.618 & 0.663 & $0.692^{*}$ & 0.642 & $0.810^{*}$ & 0.832 \\
\hline
\end{tabular}

Legenda: GAM - Generalized Additive Models, GAU - Gaussian Process, MaxEnt - Maximum Entropy, RF - Random Forest, SVM - Support Vector Machine. * modelos selecionados para o Ensemble de cada espécie.

As áreas com maior adequabilidade ambiental para a ocorrência de $H$. capillare se encontram principalmente na costa e na porção sul do Brasil, Paraguai, Bolívia, Colômbia, Equador, Costa Rica, Honduras, Guatemala, Sul do México e Chile, República Dominicana, Haiti, Porto Rico, Cuba e demais ilhas do Caribe. Algumas regiões apresentaram adequação ambiental variando de nula a média para a espécie como o norte do Chile e do México, as regiões Norte, Nordeste e Centro-Oeste do Brasil, grande parte do Peru e nos países: Argentina, Uruguai, Venezuela, Guiana, Guiana Francesa, Suriname, Panamá e El Salvador (Figura 1).

Para o modelo de $T$. tomentosum, as áreas de maior adequabilidade ambiental estão principalmente no Brasil 
(com exceção do Nordeste), sul do Paraguai, Peru, Equador, Colômbia, Venezuela, Guiana, Guiana Francesa, Suriname, Panamá, Costa rica, Nicarágua, Honduras, Guatemala, Republica Dominicana, Porto Rico e demais ilhas do Caribe. As áreas menos ambientalmente adequadas a ocorrência da espécie estão no México, Cuba, porção da Venezuela, norte do Paraguai, Uruguai, Argentina, Chile, Bolívia e na região Nordeste do Brasil (Figura 1).

A modelagem de nicho das espécies revelou algumas semelhanças entre o padrão de adequabilidade ambiental das espécies. Ambas obtiveram maior adequabilidade em áreas com alta precipitação que apresentam clima tropical ou subtropical e adequabilidade nula ou muito baixa em áreas de baixa precipitação com clima árido, semiárido ou desértico (Peel, Finlayson \& McMahon, 2007; Reboita, Gan, Rocha \& Ambrizzi, 2010). Esse aspecto está diretamente ligado a dependência de água no ambiente externo para a sobrevivência e manutenção dos processos fisiológicos nas briófitas (Vanderpoorten \& Goffinet, 2009).

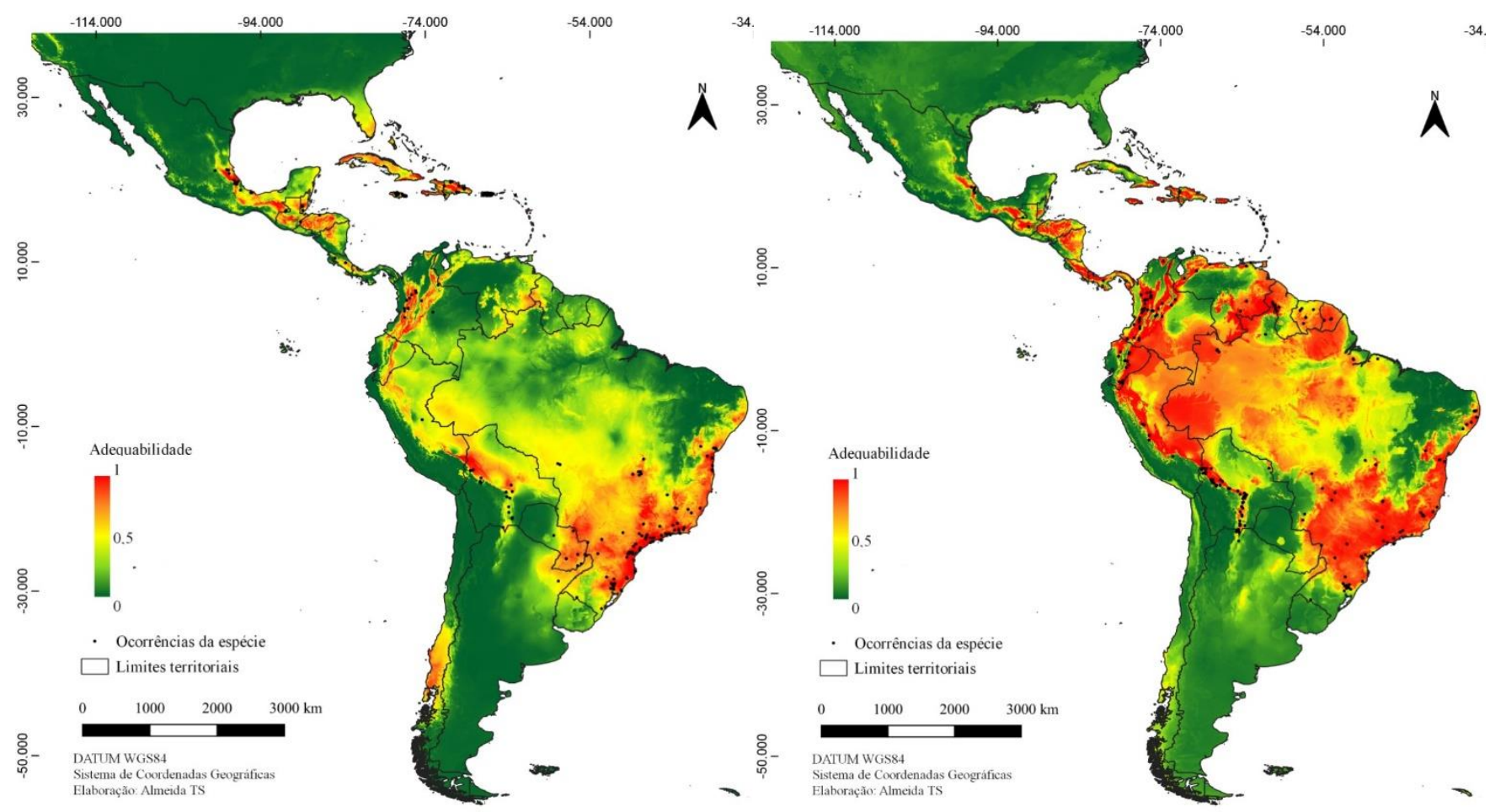

Figura 1. Mapas de modelagem de nicho ecológico das espécies Helicodontium capillare (mapa esquerdo) e Thuidium tomentosum (mapa direito).

No entanto, existem algumas diferenças entre os padrões exibidos nos modelos das espécies. A H. capillare apresentou uma adequabilidade mais restrita a ambientes com clima tropical de savana (Peel et al., 2007). Além disso, o modelo demonstrou manchas de alta adequabilidade ambiental na porção sul do Chile, região que possui precipitação anual entre 1000 e 1700 mm com clima oceânico (Peel et al., 2007; Reboita et al., 2010). Por outro lado, T. tomentosum demonstrou ser uma espécie mais tolerante, uma vez que, seu modelo apresentou alta adequabilidade ambiental também no clima tropical equatorial e tropical de monção (Peel et al., 2007).

Os países mais industrializados da América Latina são Brasil, Argentina, Colômbia, Venezuela e México (UNIDO, 2018). Desses, Brasil, Colômbia e o sul do México apresentaram alta adequabilidade ambiental para a espécie $H$. capillare e Brasil e Colômbia para T. Tometosum.

O Brasil e o México são os países que mais emitem dióxido de carbono (Global Carbon Atlas, 2019). Além disso, ambos são considerados os países mais competitivos da América Latina no âmbito industrial (UNIDO, 2018). A Colômbia é um país muito industrializado, que possui economia em ascensão graças as atividades de exportação de combustíveis fosseis e produtos agrícolas, possuindo muitos problemas ambientais (Ocampo, 2017; OEC, 2020).

No Brasil as áreas de maior adequabilidade de ambas espécies são nas regiões mais industrializadas: Sudeste e Sul, com $50,5 \%$ e $22,3 \%$ das empresas e industrias do país (IBGE, 2017). Além disso, o censo IBGE (2010a), indica que as regiões Sul e Sudeste são as mais densamente povoadas, somando 56,5\% da população do Brasil. No México, a sua capital, Cidade do México apresenta mais de 20 milhões de habitantes (IBGE, 2010b). Devido a esse exacerbado crescimento na Cidade do México e em outras cidades, existe a necessidade de monitoramento da qualidade do ar desse país (Liana et al., 2016).

Segundo Mazzoni et al. (2012) o aumento das atividades industriais e urbanização está diretamente ligada à poluição do ar por metais pesados. Sabendo dos impactos negativos que estes metais podem causar na saúde e vida da população (Muniz \& Oliveira-Filho, 2006), as espécies estudadas neste trabalho podem auxiliar no monitoramento da qualidade do 
ar nessas regiões.

A baixa susceptibilidade de ocorrência no Nordeste do Brasil, na Argentina e no Chile deve-se ao clima quente e frio desses locais (Peel et al., 2007). O aspecto do limite de ocorrência das espécie é uma das limitações para o uso de briófitas como organismos bioindicadores (Mazzoni et al., 2012). No entanto, existe uma grande diversidade neste grupo de plantas, incluindo espécies tolerantes a dessecação (Buck, 2000; Vanderpoorten \& Goffinet, 2009; Costa et al., 2010), sendo necessários novos estudos para a seleção de possíveis espécies com potencial bioindicador nas regiões citadas.

Desta forma, o uso da modelagem de nicho ecológico pode ser uma alternativa para a ampliação do conhecimento sobre este grupo de plantas com a utilização dos modelos como guia para novas expedições e aumento do esforço amostral em determinadas áreas.

\section{Conclusões}

Os nossos resultados sugerem que as espécies $H$. capillare e $T$. tomentosum possuem alta adequabilidade ambiental em regiões com alta precipitação, como também em países mais industrializados. As regiões de maior susceptibilidade também correspondem à grandes centros urbanos com altos níveis de poluição. Assim, as espécies são boas candidatas para a utilização como bioindicadoras de metais pesados nestas regiões.

\section{Referências}

Allouche, O., Tsoar, A., \& Kadmon, R. (2006). Assessing the accuracy of species distribution models: prevalence, kappa and the true skill statistic (TSS). Journal of applied ecology,43(6), 1223-1232. doi: 10.1111/j.1365-2664.2006.01214.x

Andrade, A. F. A., Velazco, S. J. E., \& Júnior, P. D. M. (2020). ENMTML: An R package for a straightforward construction of complex ecological niche models. Environmental Modelling \& Software,125, 1-11.doi: 10.1016/j.envsoft.2019.104615

Bagliano, R. V. (2012). Principais organismos utilizados como bioindicadores relatados com uso de avaliadores de danos ambientais. Revista Meio Ambiente e Sustentabilidade, 2(1), 24-40. Recuperado de https://www.uninter.com/revistameioambiente/index.php/meioAmbiente/ article/view/113/50

Barbet-Massin, M., Jiguet, F., Albert, C. H., \& Thuiller, W. (2012). Selecting pseudo-absences for species distribution models: how, where and how many?. Methods in ecology and evolution, 3(2), 327-338. doi: 10.1111/j.2041-210X.2011.00172.x

Barbosa, S. F., \& Carvalho, M. A. S. (2016). Análise Cienciométrica da Utilização de Briófitas como Bioindicadores. Caderno de Pesquisa, 28(1), 34-47. doi: $10.17058 /$ cp.v28i1.4596

Breiman, L. (2001). Random forests. Machine learning, 45(1), 5-32.

Buck, W. R. (2000). Morphology and Classification of Mosses. In: J. A. Shaw \& B. Goffinet (eds.). Bryophyte Biology. (1a ed., cap. 13, pp. 71-123). Cambridge: Cambridge University Press.

Câmara, P. E, Teixeira, R., Lima, J., \& Lima, J. (2003). Musgos urbanos do Recanto das Emas, Distrito Federal, Brasil. Acta botanica brasilica, 17(4), 507-513. doi: 10.1590/S0102-33062003000400003

Costa, D. P., Camara, P. E. A. S., Porto, K.C.; Luizi-Ponzo, A.P \& IlkiuBorges, A.L.( 2010). Musgos. In: Catálogo de Plantas e Fungos do Brasil. v.1. (1a ed., pp. 452-521). Rio de Janeiro: Andrea Jakobsson Estúdio, Instituto de Pesquisas Jardim Botânico do Rio de Janeiro. Recuperado de https://static.scielo.org/scielobooks/z3529/pdf/forzza9788560035083.pdf

Dormann, C.F., Elith, J., Bacher, S., Buchmann, C., Carl, G., Carre, G., Garcia Marquez, J.R., Gruber, B., Lafoourcade, B., Leitao, P.J., Münkemüller, T., Mcclean, C., Osborne, P.E., Reineking, B., Schröder, B., Skidmore,
A.K., Zurell, D. \& Lautenbach, S. (2013). Collinearity: a review of methods to deal with it and a simulation study evaluating their performance. Ecography, 36(1), 27-46. doi: 10.1111/j.16000587.2012.07348.x

Fick, S. E. \& Hijmans, R. J. (2017) WorldClim 2: new 1-km spatial resolution climate surfaces for global land areas. International journal of climatology, 37(12), 4302-4315. doi: 10.1002/joc.5086

Frahm, J.P. (1991). Dicranaceae: Campylopodioideae, Paraleucobryoideae. Flora Neotropica 54: 1-238.

GBIF. (2020). Global Biodiversity Information Facility. Disponível em: https://www.gbif.org/

GCA. (2020). Global Carbon Atlas. Disponível em: http://www.globalcarbonatlas.org/en/CO2-emissions.

Goffinet, B. \& Shaw, A. J. (2009) Bryophyte Biology. (2a ed.). Cambridge: Cambridge University Press

Golding, N., \& Purse, B. V. (2016). Fast and flexible Bayesian species distribution modelling using Gaussian processes. Methods in Ecology and Evolution, 7(5), 598-608. doi: https://doi.org/10.1111/2041210X.12523

Guisan, A., Edwards Jr, T. C., \& Hastie, T. (2002). Generalized linear and generalized additive models in studies of species distributions: setting the scene. Ecological modelling, 157(2-3), 89-100. Recuperado de: https://web.stanford.edu/ hastie/Papers/GuisanEtAl_EcolModel2003.pdf

Guisan, A., \& Zimmermann, N. E. (2000) Predictive habitat distribution models in ecology. Ecological modelling, 135 (2-3), 147-186. doi: 10.1016/S0304-3800(00)00354-9

IBGE. (2010a). Censo 2010. Disponível em: https://censo2010.ibge.gov.br

IBGE (2010b). Atlas do Censo Demográfico 2010. Recuperado de: https://biblioteca.ibge.gov.br/visualizacao/livros/liv64529_cap1.pdf.

IBGE. (2017). Demografia das empresas 2015. Rio de Janeiro, 91p. Disponível https://www.ibge.gov.br/estatisticas/economicas/industria/9068demografia-das empresas.html?=\&t=publicacoes.

Inácio-Silva, M.; Carmo, D. M., \& Peralta, D. F. (2017) As espécies brasileiras endêmicas de Campylopus Brid.(Bryophyta) estão ameaçadas? Uma análise usando modelagem para avaliar os seus estados de conservação. Hoehnea, 44 (3), 464-472. doi: 10.1590/22368906-08/2017

Liana, M., Edison, O., Jorge, M., Marcos, M., Leda, A., \& Guerrero, U. (2016) Situação atual da qualidade do ar das principais cidades da América Latina. Ciência e Natura, 38, 523-531. doi: 10.5902/2179460X20290

Mazzoni, A. C., Lanzer, R., Bordin, J., Schafer, A., \& Wasum, R. (2012) Mosses as indicators of atmospheric metal deposition in an industrial area of southern Brazil. Acta Botanica Brasilica, 26(3), 553-558. doi: $10.1590 / \mathrm{S} 0102-33062012000300005$

Muniz, D.H.F. \& Oliveira-Filho, E.C. (2006). Metais pesados provenientes de rejeitos de mineração e seus efeitos sobre a saúde e o meio ambiente. Universitas: ciências da saúde, 4(1), 83-100. doi: 10.5102/ucs.v4i1.24

OEC. (2020). Observatorio de Complejidad Económica. Recuperado de: https://atlas.media.mit.edu/es/profile/country/col/

Ocampo, J. S. (2017). Vivienda industrializada: antecedentes en el mundo y propuesta al déficit de vivienda social en Colombia. Cuadernos de Vivienda y urbanismo, 10(20), 79-96.doi: 10.11144/Javeriana.cvu1020.viam

Peel, M. C., Finlayson, B. L., \& McMahon, T. A. (2007). Updated world map of the Köppen-Geiger climate classification. Hydrology and Earth System Sciences, 11, 1633-1644. doi: 10.5194/hess-11-1633-2007

Phillips, S. J., Anderson, R. P., \& Schapire, R. E. (2006). Maximum entropy modeling of species geographic distributions. Ecological modelling, 190(3-4), 231-259. doi: 10.1016/j.ecolmodel.2005.03.026

R Core Team. (2020). R: A language and environment for statistical computing. R Foundation for Statistical Computing, Vienna, Austria. Version 4.0.0. Disponível em: https://www.R-project.org

SpeciesLink. (2020). SpeciesLink. Disponível em: http://www.splink.org.br/. 
Reboita, M. S., Gan, M. A., Rocha, R. P. D., \& Ambrizzi, T. (2010). Regimes de precipitação na América do Sul: uma revisão bibliográfica. Revista brasileira de meteorologia,25(2), 185-204. doi: 10.1590/S010277862010000200004.

Salcedo-Sanz, S., Rojo-Álvarez, J. L., Martínez-Ramón, M., \& Camps-Valls, G. (2014). Support vector machines in engineering: an overview. Wiley Interdisciplinary Reviews: Data Mining and Knowledge Discovery, 4(3), 234-267. doi: 10.1002/widm. 1125

Shepherd, G. J. (2005). Plantas terrestres. In. Lewinsohn, T. M. (Org.). Avaliação do estado do conhecimento da biodiversidade brasileira. (1a ed, pp. 145-192). Série Biodiversidade vol 2. Ministério do Meio Ambiente.

Silva, M. P. P., Kamino, L. H. Y., \& Pôrto, K. C. (2014). Is the current network system of protected areas in the Atlantic Forest effective in conserving key species of bryophytes? Tropical Conservation Science, 7, 61-74. doi: 10.1177/194008291400700110

Teixeira, D.C.L.; Moreira, I.F.V.; Coelho, M.A.; Amaral, Y.F.Q. \& Cupertino, M.C. (2020). Exposição a contaminantes ambientais inorgânicos e danos à saúde humana. Brazilian Journal of Health Review, 3 (4), 10353-10369. doi: $10.34119 /$ bjhrv3n4-256

UNIDO. (2018). Competitive Industrial Performace Report 2018. Bienal cip report. $116 \mathrm{p}$.

Vanderpoorten, A. \& Goffinet, B. (2009). Introduction of Bryophytes. Cambridge: Cambridge University Press.

Velazco, S. J. E., Villalobos, F., Galvão, F., \& De Marco Júnior, P. (2019). A dark scenario for Cerrado plant species: Effects of future climate, land use and protected areas ineffectiveness. Diversity and Distributions, 25(4), 660-673. doi: 10.1111/ddi.12886

Vieira, H. C. A., Oliveira, R. R., Silva, M. L. A., Silva, D. L. S., da Conceição, G. M., \& Oliveira, H. C. (2017). Briófitas de ocorrências em São João do Sóter, Maranhão, Brasil. Acta Brasiliensis, 1(2), 8-12. doi: 10.22571/Actabra12201745

Zurell, D., Zimmermann, N. E., Gross, H., Baltensweiler, A., Sattler, T., \& Wüest, R. O. (2020). Testing species assemblage predictions from stacked and joint species distribution models. Journal of Biogeography, 47(1), 101-113. doi: 10.1111/jbi.13608

Este artigo foi publicado com acesso aberto para distribuíção sob os termos do Licença de Atribuição Creative Commons, que permite uso irrestrito, distribuição, e reprodução em qualquer meio, desde que o trabalho original seja devidamente citado. 